\title{
DENSIDADE BÁSICA E RESISTÊNCIA MECÂNICA DE MADEIRAS DE RESÍDUOS FLORESTAIS DA REGIÃO AMAZÔNICA
}

\author{
Letícia da Silva Moreira'; Victor Hugo Pereira Moutinho². \\ 1Universidade Federal do Oeste do Pará, Santarém - Pará, Brasil, moreira.leticia94@gmail.com \\ Universidade Federal do Oeste do Pará, Santarém - Pará, Brasil, victor.ctmadeira@gmail.com
}

RESUMO: A madeira da copa que permanece na floresta após a colheita pode apresentar diversas aplicações como em movelaria, estruturas e artefatos, desde que conhecidas suas propriedades tecnológicas. Este trabalho visou caracterizar a densidade básica e propriedades mecânicas da madeira de resíduos florestais. Na área de concessão florestal da Gleba Nova Olinda foram coletadas madeira de galho e base do fuste para caracterização realizada segundo as recomendações da norma utilizada. Os resultados foram analisados através do teste $T$. A densidade básica foi equivalente a 0,81 e 0,94 $\mathrm{g} \mathrm{cm}^{-3}$ para Manilkara sp. e 0,91 e 0,88 $\mathrm{g} \mathrm{cm}^{-3} \mathrm{em}$ Hymenaea sp., para a madeira de galho e base do tronco, respectivamente, os quais não diferiram estatisticamente entre si quando comparadas as médias de Manilkara sp., enquanto em Hymenaea sp. não houve diferença estatística para essa propriedade. A resistência mecânica da madeira de galho e base do fuste de Manilkara sp. não apresentou diferença estatística significativa, a exceção do teste de dureza Janka plano tangencial e transversal. Os ensaios de Hymenaea sp. revelaram propriedades mecânicas estatisticamente distintas para a madeira de galho e base do fuste, exceto em compressão paralela às fibras, onde os módulos de resistência foram equivalentes a 85 e $94 \mathrm{MPa}$ para galho e base do tronco, respectivamente. A madeira de resíduos das espécies caracterizadas apresentou alta densidade e resistência mecânica semelhante à da base do fuste e a utilização desse material representa potencialidade para obtenção de produtos com valor agregado, desde que consideradas suas particularidades e estabilidade dimensional.

PALAVRAS-CHAVE: Caracterização mecânica, Galhos, Propriedades físicas.

\section{SPECIFIC GRAVITY AND MECHANICAL RESISTANCE OF FOREST LEFTOVER FROM THE AMAZON REGION}

ABSTRACT: The leftover timber that remains in the forest after harvesting may present several applications as in furniture, structures and artifacts, if their technological properties are known. This work aimed to characterize the basic density and mechanical properties of forest leftover wood. At the area of forest concession of the Gleba Nova Olinda it was collected leftover and base stem wood for characterization accomplished according to the recommendations of the standard used. The results 
were analyzed using the T-test. The basic density was 0.81 and $0.94 \mathrm{~g} \mathrm{~cm}^{-3}$ for Manilkara sp. and 0.91 and $0.88 \mathrm{~g} \mathrm{~cm}^{-3}$ in Hymenaea sp., respectively, for leftover wood and base stem, which did not differ statistically from each other when comparing the means of Manilkara sp., while in Hymenaea sp. there was no statistical difference for this property. The mechanical resistance of the leftover wood and the base stem of Manilkara sp. did not present significant statistical difference, except for the tangential and transverse plane in the Janka hardness test. The Hymenaea sp. revealed statistically different mechanical properties for twig and base wood, except for compression parallel to the fibers, where the resistance was equivalent to 85 and 94 MPa for leftover and base stem wood, respectively. The wood of residues of the species characterized presented high density and mechanical resistance similar to its base stem and the use of this material represents the potential for obtaining products with added value, considering their particularities and dimensional stability.

KEYWORDS: Forest leftover wood, Mechanical characterization, Physical properties.

\section{DENSIDAD BÁSICA Y RESISTENCIA MECÁNICA DE MADERAS DE RESIDUOS FORESTALES DE LA REGIÓN AMAZÓNICA}

RESUMEN: La madera de la copa que permanece en el bosque después de la extracción maderera puede presentar diversas aplicaciones como en movelaria, estructuras y artefactos, desde que conocidas sus propiedades tecnológicas. Este trabajo pretendía caracterizar la densidad básica y las propiedades mecánicas de la madera de residuos forestales. En el área de concesión forestal de la Gleba Nova Olinda fueron colectadas madera de rama y base del fuste para caracterización realizada según las recomendaciones de la norma utilizada. Los resultados fueron analizados a través del test T. La densidad básica fue equivalente a 0,81 y 0,94 $\mathrm{g} \mathrm{cm}^{-3}$ para Manilkara sp. y 0,91 y 0,88 $\mathrm{g} \mathrm{cm}^{-3}$ en Hymenaea sp., para la madera de rama y base del tronco, respectivamente, los cuales no diferían estadísticamente entre sí cuando se compararon las medias de Manilkara sp., mientras que en Hymenaea sp. no hubo diferencia estadística para esa propiedad. La resistencia mecánica de la madera de gajo y base del fuste de Manilkara sp. no presentó diferencia estadística significativa, la excepción de la prueba de dureza Janka plano tangencial y transversal. Los ensayos de Hymenaea sp. revelaron propiedades mecánicas estadísticamente distintas para la madera de gajo y base del fuste, excepto en compresión paralela a las fibras, donde los módulos de resistencia fueron equivalentes a 85 y 94 MPa para gajo y base del tronco, respectivamente. La madera de residuos de las especies caracterizadas presentó alta densidad y resistencia mecánica similar a de la base del fuste y la utilización de ese material representa potencial para la obtención de 
productos con valor agregado, siempre que se consideren sus particularidades y estabilidad dimensional.

PALABRAS CLAVE: Caracterización mecánica, Gajos, Propiedades físicas.

\section{INTRODUÇÃO}

A floresta amazônica abriga imensa diversidade de espécies e fornecem diversos produtos, os quais necessitam ser utilizados por meio de práticas sustentáveis (KANASHIRO, 2014). A realização de estudos sobre a respectiva tecnologia de seus produtos fornece parâmetros técnicos que possibilitem o seu aprimoramento e valorização de seus subprodutos podendo assim contribuir com a sustentabilidade das intervenções na floresta utilizando alternativas sustentáveis como o aproveitamento de resíduos florestais (BAGGIO; CARPANEZZI, 1995).

Segundo Braz et al. (2014), o baixo rendimento na colheita e atividade madeireira, especialmente das empresas da região Amazônica, ocorre principalmente devido à escassez de conhecimento da potencialidade para diferentes aplicações que os resíduos florestais podem oferecer. Barbosa et al. (2001) afirmam que o uso mais nobre de resíduos madeireiros certamente traria vantagens econômicas ao possibilitarem a produção de novos produtos com maior valor agregado, além de vantagens sociais, como a geração de empregos com o surgimento das atividades decorrentes de sua aplicação.

O material conhecido como resíduo florestal é aquele que permanece na floresta após a colheita (PULITO; JUNIOR, 2009). Ao acompanhar o processo de colheita de árvores nas áreas de manejo, nota-se que os galhos e a madeira da copa das árvores permanecem na floresta e, se tratando de espécies florestais da Amazônia, em que as árvores são em sua grande maioria de grande porte, o volume de madeira da copa é significativo (PONTES et al., 2012). Silva-Ribeiro 
(2013) ao estudar o volume de resíduos florestais de espécies arbóreas na Floresta Nacional do Tapajós, verificou significativas proporções destes em relação ao fuste, em que a cada metro cúbico de fuste retirado da floresta, permanecia aproximadamente 0,45 $\mathrm{m}^{3}$ de galho com diâmetro acima de 30 cm, o qual pode ter usos mais nobres, desde que conhecida suas respectivas propriedades tecnológicas.

De acordo com SEMAS (2016), somente a mesorregião do Baixo Amazonas foi responsável pelo fornecimento próximo a três milhões de metros cúbicos de madeira em tora entre 2008 e 2012, levando então a considerar a volumetria mínima de 1,3 milhão de metros cúbicos de resíduos florestais aptos ao desdobro. Nisto, vale destacar as espécies popularmente conhecidas maçaranduba e jatobá as quais, segundo SFB (2016), encontram-se entre as mais comercializadas e valorizadas no país. Nesse contexto, torna-se imprescindível conhecer as propriedades tecnológicas da madeira residual desse grupo de espécies principais, visando possibilitar sua aplicação e inserção no mercado.

A avaliação do potencial tecnológico da madeira - neste caso, resíduos - com disponibilidade comercial se procede a partir da determinação de suas propriedades físico-mecânicas, as quais são fundamentais para definir adequadamente a aplicação da madeira às diversas utilizações, principalmente aquelas que requerem maior resistência (ARAÚJO, 2007). Cada espécie possui diferentes características, se tornando também necessária a verificação dos diversos fatores que afetam intrinsicamente em sua qualidade de uso (GONÇALVES et al., 2009a; PIGOZZO, 2009; GONÇALVES; LELIS, 2012; ALMEIDA et al., 2013).

Segundo Sette Jr et al. (2012), a densidade é um dos parâmetros mais importantes entre as diversas propriedades da madeira, pois sua variação influencia as demais características tecnológicas desta. Entre 
essas propriedades, a que se destaca como de fundamental importância para seu emprego tecnológico é a densidade básica, visto que apresenta importante correlação com as diversas outras propriedades da madeira (BATISTA et al. (2010); SANTOS; SANSíGOLO, 2007). Lobão et al. (2004) afirmam que a densidade tem influência direta nas propriedades mecânica da madeira e sua variação pode ser estimada com base na variabilidade da densidade.

Para Logsdon et al. (2007) e Miranda et al. (2012), as propriedades mecânicas são importantes para avaliar a aplicação e qualidade do material pois fornece parâmetros indicadores de resistência como o módulo de ruptura (MOR), o módulo de elasticidade (MOE), os quais subsidiam avaliações em relação às principais propriedades mecânicas, citadas por Beltrame et al. (2010), tais como a resistência ao esforço de compressão paralela às fibras, flexão estática, cisalhamento e o teste de dureza Janka. Estes módulos são parâmetros de grande importância na caracterização tecnológica da madeira, pois ambos estimam a resistência do material submetido a uma força aplicada (LEONELLO et al., 2012).

o conhecimento dessas características tecnológicas pode viabilizar, segundo Braz et al. (2014), a inserção desse tipo de material no mercado e, assim, por meio do melhor aproveitamento da árvore, contribuir para a viabilidade econômica e sustentabilidade das intervenções na floresta, visando o manejo que assegure a conservação das florestas amazônicas. Os diversos trabalhos relacionados ao aproveitamento de resíduos encontrados em literatura têm como foco a utilização da madeira descartada durante o processamento em serrarias e marcenarias (DUTRA, 2005; FEITOSA, 2008; GONÇALVES et al., 2009b; PEREIRA et al., 2011; ZAU et al., 2014). Nesse sentido, o objetivo deste trabalho foi realizar a caracterização física e mecânica da madeira proveniente de resíduos florestais. 


\section{MATERIAL E MÉTODOS}

Com base no levantamento de dados com as informações disponibilizadas pela Secretaria Estadual do Meio Ambiente e Sustentabilidade (SEMAS) via SISFLORA, foram obtidos dados referentes ao período de 2009 a 2015 e, a partir disso, estimando-se a volumetria das espécies comercializadas na mesorregião do Oeste do Pará. Os valores foram transcritos no software Excel For Windows ${ }^{\circledR}$, viabilizando quantificar características como volumetria das espécies, valor por metro cúbico, agrupamento, localidade da colheita. Baseado nisto, foram selecionadas espécies de maior expressividade comercial a citar: maçaranduba (Manilkara sp.) e jatobá (Hymenaea sp.).

O material foi proveniente da área de florestas públicas estaduais integrante da Gleba Nova Olinda, a qual possui área total de cerca de 87000 hectares, sendo integrante do conjunto de glebas Mamuru-Arapiuns, localizada no eixo do Rio Arapiuns, a distância de 140 km de Santarém (Figura 1), situada próxima à comunidade Cachoeira do Aruã, região oeste do estado do Pará (IDEFLOR, 2010).

As árvores já haviam sido identificadas e localizadas para o arraste da tora na atividade de manejo, baseado nos mapas de corte nas respectivas unidades de trabalho. Por conta disso, era conhecido a área onde os galhos permaneceram. No local, a equipe analisou a direção de queda da árvore para localizar os seus respectivos galhos.

Foi coletado material de quatro árvores por espécie, seguindo o critério de galhos com DAP $\geq 50 \mathrm{~cm}$ a partir da primeira bifurcação, de onde foram retirados pranchões centrais in loco. Posteriormente, os mesmos foram seccionados com motosserra em sarrafos obtidos do cerne, com dimensões mínimas de $10 \mathrm{~cm} \times 10 \mathrm{~cm} \times$ $200 \mathrm{~cm}$, considerando largura, espessura e comprimento, respectivamente. Foi coletado também 
material da base do fuste para fim de comparação com as propriedades do galho.

Os sarrafos foram submetidos ao desdobro secundário na marcenaria Paraná localizada na comunidade Tabocal, em Santarém, oeste do Pará, sendo seccionados em corpos de prova reduzidos com dimensões seguindo as recomendações da norma American Society for Testing and Materials - ASTM D 143 - 14 (2009), para possibilitar os ensaios físicomecânicos da madeira.

A densidade básica, obtida pela razão entre massa seca e volume saturado, foi obtida de acordo com os procedimentos descritos pela norma American Society for Testing and Materials - ASTM D 143 - 14 (2009), com o número mínimo de 8 amostras por indivíduo tanto para material de galho quanto da base do fuste. As medidas de massa foram realizadas em balança eletrônica de 0,01 g de sensibilidade. As dimensões foram obtidas com auxílio de paquímetro digital de 0,01 $\mathrm{mm}$ de precisão. 0 método de determinação do volume foi adaptado pois não foi possível realizar a imersão em mercúrio recomendado pela norma, assim, procedeu-se a o método esteriométrico para obtenção do volume.

Os ensaios mecânicos de compressão paralela às fibras, flexão estática, cisalhamento e dureza Janka foram realizados em uma máquina universal EMIC modelo DL 30000, com sistema de obtenção de dados automatizado, localizada no Laboratório de Tecnologia da Madeira da Universidade Federal do Oeste do Pará. A confecção dos corpos de prova e os ensaios mecânicos foram realizados segundo as diretrizes preconizadas pela norma ASTM D143 14 (2009). A amostragem foi composta pelo número mínimo de 10 corpos de prova de cada indivíduo para cada teste mecânico. Os resultados obtidos foram corrigidos para a umidade de referência de 12\%, segundo as equações 1 e 2 prescritas pela norma. 
Para correção da resistência:

$$
f_{12}=f_{u \%}\left[\frac{1+3(U \%-12)}{100}\right]
$$

Em que:

$f_{12}=$ valor da resistência no teor de $12 \%$ de umidade

$f_{U \%}=$ valor da resistência encontrado no teor de umidade ensaiado; $\mathrm{e}$

$\mathrm{U} \%$ = umidade do corpo-de-prova no momento do ensaio.

Para a rigidez:

$$
E_{12}=E_{u \%}\left[\frac{1+2(U \%-12)}{100}\right]
$$

Em que:

$E_{12}=$ módulo de elasticidade no teor de 12\% de umidade

$\mathrm{E}_{\mathrm{U} \%}$ = módulo de elasticidade encontrado no teor de umidade ensaiado; $\mathrm{e}$

$\mathrm{U} \%$ = umidade do corpo-de-prova no momento do ensaio.

Para a avaliação resultados e comparação das médias obtidas, foram realizados os testes de Shapiro-Wilk e de Bartlett, seguido pelo teste $\mathrm{T}$ em nível de $5 \%$ de probabilidade de erro ( $p>0,05)$, para os dados com distribuição normal ( $p<0,05)$. As análises foram processadas no programa RStudio (2016).

Os resultados obtidos para 0 material estudado foram comparados com médias das propriedades da madeira de fuste principal e com aqueles existentes em literatura para as mesmas espécies estudadas, conforme suas respectivas caracterizações de propriedades mecânicas e densidade básica.

\section{RESULTADOS E DISCUSSÃO}

Os valores médios encontrados para densidade básica madeira proveniente de galho e da base do fuste de Hymenaea sp. foram equivalentes a 0,81 e 0,94 $\mathrm{g} \mathrm{cm}^{-3}$, respectivamente, os quais apresentam diferença estatisticamente significativa. Para Manilkara sp. foram obtidos valores médios de 0,91 e 0,88 $\mathrm{g} \mathrm{cm}^{-3}$ para galho e da base do fuste, 
respectivamente, não sendo detectada diferença estatística significativa, como apresentado na Tabela 1, onde também constam o módulo de resistência a compressão paralela às fibras e módulos de resistência e elasticidade na flexão estática da madeira de galho e fuste registrados, corrigidos à $12 \%$ de umidade.

Tabela 1. Densidade básica, resistência a compressão paralela às fibras e módulos de resistência e elasticidade a flexão estática de galho e fuste das espécies estudadas.

\begin{tabular}{|c|c|c|c|c|c|c|c|c|}
\hline \multirow{3}{*}{ Espécie } & \multirow{2}{*}{\multicolumn{2}{|c|}{$\rho_{\text {bas }}\left(\mathrm{g} \mathrm{cm}^{-3}\right)$}} & \multicolumn{2}{|c|}{$f_{c 0}$} & \multicolumn{2}{|c|}{$f_{M}$} & \multicolumn{2}{|c|}{$\mathrm{E}_{\mathrm{MO}}$} \\
\hline & & & \multicolumn{2}{|c|}{ (MPa) } & \multicolumn{2}{|c|}{$(\mathrm{MPa})$} & \multicolumn{2}{|c|}{ (GPa) } \\
\hline & $G$ & $F$ & $G$ & $F$ & $G$ & $F$ & $G$ & $\mathrm{~F}$ \\
\hline Hymenaea sp. & $\begin{array}{c}0,81 \\
(3,93)\end{array}$ & $\begin{array}{l}0,94^{*} \\
(9,57)\end{array}$ & $\begin{array}{l}85,01 \\
(14,01)\end{array}$ & $\begin{array}{l}94,25 \text { * } \\
(16,66)\end{array}$ & $\begin{array}{l}101,75 \\
(24,92)\end{array}$ & $\begin{array}{l}153,32 \text { * } \\
(21,62)\end{array}$ & $\begin{array}{c}11,01 \\
(27,64)\end{array}$ & $\begin{array}{l}18,47 \text { * } \\
(19,55)\end{array}$ \\
\hline Manilkara sp. & $\begin{array}{c}0,91 \\
(2,37)\end{array}$ & $\begin{array}{c}0,88 \mathrm{~ns} \\
(2,35)\end{array}$ & $\begin{array}{l}69,42 \\
(16,02) \\
\end{array}$ & $\begin{array}{c}71,01 \mathrm{~ns} \\
(7,60)\end{array}$ & $\begin{array}{l}125,26 \\
(26,46) \\
\end{array}$ & $\begin{array}{c}137,18 \mathrm{~ns} \\
(36,64)\end{array}$ & $\begin{array}{c}15,14 \\
(18,35) \\
\end{array}$ & $\begin{array}{c}14,84 \text { ns } \\
(23,73)\end{array}$ \\
\hline
\end{tabular}

$\rho_{\text {bas }}=$ densidade básica; $f_{c 0}=$ resistência a compressão paralela às fibras; $f_{M}=$ módulo de resistência na flexão estática; $\mathrm{E}_{\mathrm{MO}}$ = módulo de resistência e elasticidade a flexão estática. Valores entre parênteses indicam o coeficiente de variação (\%); $G=$ Galho; $F=$ Fuste; GPa: gigaPascal. * = coeficiente significativo; $n s$ = diferença não significativa de acordo com o teste $T$ à $5 \%$ de significância $(p>0,05)$ na mesma linha para cada propriedade.

Os valores de densidade básica encontrados para galho e fuste da madeira das espécies estudadas (Tabela 1) são considerados altos, segundo classificação de IPT (2013). Para a madeira proveniente de galho de Manilkara sp. foi obtido valor médio de densidade superior ao registrado por Silveira et al. (2013) de 0,795 g cm3 para galhos grossos (diâmetro $>10$ $\mathrm{cm})$ e $0,877 \mathrm{~g} \mathrm{~cm}^{-3}$ para a base do tronco de Manilkara huberi. Gonçalez et al. (2002) encontrou valor semelhante de $0,94 \mathrm{~g} \mathrm{~cm}^{-3}$ para a densidade básica de Manilkara sp. ao estudar as características tecnológicas de madeiras amazônicas provenientes da região de Tucuruí - PA.

Conforme os resultados da Tabela 1, pode ser observada menor densidade para madeira proveniente de galhos em Hymenaea sp. Estes valores foram 
superiores aos encontrados por Bergman et al. (2010) de 0,71 $\mathrm{g} \mathrm{cm}^{-3} \mathrm{e}$ por Souza et al. (2002) de 0,76 $\mathrm{g} \mathrm{cm}^{-3}$ para fuste de Hymenaea courbaril. Por outro lado, os mesmos foram inferiores aos encontrados por Lahr et al. (2016) de $0,91,1,05$ e $1,08 \mathrm{~g} \mathrm{~cm}^{-3}$ para $H$. courbaril proveniente de diferentes regiões do norte do país.

O comportamento dessemelhante entre a densidade da madeira da copa e do fuste, como observado em Hymenaea sp. pode ser influenciado por diferenças nas suas características anatômicas, as quais podem ser configuradas por uma maior espessura de parede celular ou de um aumento na proporção das fibras em relação, por exemplo, à proporção de vasos (OLIVEIRA; SILVA, 2003), resultando em crescimento da densidade e, deste modo, diferentes comportamentos de estabilidade dimensional e resistência mecânica (LATORRACA; ALBUQUERQUE, 2000). Assim como ser influenciada pela idade das árvores, as quais não se tem conhecimento. Gonçalves et al. (2009a) discorre sobre o aumento da densidade da madeira com a idade das árvores, como resultado das modificações das células do câmbio, formando o lenho adulto.

Os resultados dos ensaios mecânicos apontam semelhança entre as propriedades de galho e base do fuste de Manilkara sp., havendo diferença somente entre as médias obtidas para dureza Janka no plano tangencial e transversal. Em relação a resistência a compressão paralela às fibras de Manilkara sp., como apresentado na Tabela 1, esta foi menor para a madeira de densidade superior. Isso pode ser atribuído também as suas características anatômicas. Segundo Cardoso (2015), madeiras de maior frequência e espessura de raio e com paredes menos espessas estão associadas a um menor módulo de ruptura no ensaio de compressão paralela às fibras.

As madeiras de galho e base do fuste de Hymenaea sp. apresentaram diferença estatisticamente significativa entre os valores médios encontrados para as propriedades mecânicas, com 
exceção da resistência ao cisalhamento obtido para a madeira de galho o qual foi equivalente à da base do fuste da espécie (Tabela 2).

Tabela 2. Resultados da resistência ao cisalhamento e dureza Janka para as espécies ensaiadas

\begin{tabular}{|c|c|c|c|c|c|c|c|c|}
\hline \multirow{3}{*}{ Espécie } & \multicolumn{2}{|c|}{$f_{v 0}$} & \multicolumn{2}{|c|}{$f_{t g}$} & \multicolumn{2}{|c|}{$f_{t v}$} & \multicolumn{2}{|c|}{$f_{\text {rad }}$} \\
\hline & \multicolumn{2}{|c|}{$(\mathrm{MPa})$} & \multicolumn{2}{|c|}{$(k N)$} & \multicolumn{2}{|c|}{$(\mathrm{kN})$} & \multicolumn{2}{|c|}{$(k N)$} \\
\hline & G & $F$ & G & $\mathrm{F}$ & $G$ & $\mathrm{~F}$ & G & $\mathrm{F}$ \\
\hline Hymenaea sp. & $\begin{array}{c}18,17 \\
(17,04)\end{array}$ & $\begin{array}{c}17,85 \mathrm{~ns} \\
(17,69)\end{array}$ & $\begin{array}{c}11,20 \\
(17,31)\end{array}$ & $\begin{array}{l}16,40 \text { * } \\
(17,83)\end{array}$ & $\begin{array}{c}14,0 \\
(12,43)\end{array}$ & $\begin{array}{l}16,70 \text { * } \\
(16,78)\end{array}$ & $\begin{array}{c}12,40 \\
(12,92)\end{array}$ & $\begin{array}{l}16,60 \text { * } \\
(21,07)\end{array}$ \\
\hline Manilkara sp. & $\begin{array}{c}17,22 \\
(22,58) \\
\end{array}$ & $\begin{array}{c}15,96 \text { ns } \\
(21,87)\end{array}$ & $\begin{array}{c}12,60 \\
(15,35)\end{array}$ & $\begin{array}{l}14,30 \text { * } \\
(10,03)\end{array}$ & $\begin{array}{l}13,80 \\
(6,47) \\
\end{array}$ & $\begin{array}{l}11,50 \text { * } \\
(11,09)\end{array}$ & $\begin{array}{c}13,10 \\
(26,50) \\
\end{array}$ & $\begin{array}{c}13,30 \mathrm{~ns} \\
(18,08)\end{array}$ \\
\hline
\end{tabular}

$\overline{\mathrm{f}_{\mathrm{vo}}=}$ resistência ao cisalhamento; $\mathrm{f}_{\mathrm{tg}}=$ dureza Janka transversal; $\mathrm{f}_{\mathrm{gg}}=$ dureza Janka tangencial; $\mathrm{f}_{\mathrm{rad}}=$ dureza Janka radial; GPa: gigaPascal; Valores entre parênteses indicam o coeficiente de variação $(\%)$ * = coeficiente significativo; $n s$ = diferença não significativa de acordo com o teste Tà 5\% de significância $(p>0,05)$ na mesma linha para cada propriedade.

O valor médio registrado para galho e fuste de Manilkara sp. para os ensaios de compressão paralela às fibras (Tabela 1) foram inferiores aos encontrados por Souza et al. (2002) de 108,76 MPa. Esse comportamento pode estar relacionado a diferentes espécies do gênero Manilkara, assim como diferentes densidades da madeira.

O módulo de elasticidade médio para a o ensaio de flexão estática foi semelhante ao encontrado por Souza et al. (2002), equivalente a 13,8 GPa. Rosa et al. (2014) encontrou 19,99 GPa para o mesmo teste à $12 \%$ de umidade. Os valores obtidos para galho e fuste da madeira de Manilkara sp. para o teste de resistência ao cisalhamento foram superiores ao de Souza et al. (2002) que encontraram 64,8 MPa neste tipo de ensaio para maçaranduba. Segundo Gomes et al., (2005), a madeira de maçaranduba é considerada de alta durabilidade em contato com o solo, sendo indicada para utilização em postes, cercas, assoalhos entre diversos outros usos. 
Os ensaios mecânicos de Hymenaea sp. revelaram valores médios estatisticamente distintas para as propriedades mecânicas de galho e fuste principal, sendo semelhantes somente os resultados para o teste de cisalhamento. A principal evidência para este comportamento mecânico diferente entre as madeiras testadas pode ser atribuída às diferentes densidades entre as duas porções da árvore para esta espécie que podem ser resultados de diferente característica anatômica ou teor de extrativos mais elevado. Evans et al. (2000) afirmam que as variações no tamanho dos poros e na espessura das paredes celulares tornam uma madeira detentora de maior quantidade de matéria por unidade de volume do que outra e, portanto, maior densidade.

Os valores médios verificados para Hymenaea sp. nos testes de compressão paralela às fibras foram maiores que o encontrado por Araújo (2007) para Hymenaea courbaril, equivalente a 75,81 MPa. No estudo de Dias e Lahr (2004), ao trabalharem com a madeira de Hymenaea sp., foi observado um valor superior aos obtidos, correspondente a $91 \mathrm{MPa}$ para o mesmo teste de resistência mecânica.

Em relação a dureza determinada pelo método Janka, os resultados obtidos para o material de galho e fuste nos planos normal (transversal) e paralelo às fibras (tangencial e radial) e apresentam valores de resistência com razão aproximada a 1, sendo este um resultado coerente com o obtido por Lahr et al. (2010). Silva Filho et al. (1992) avaliaram em seu trabalho uma proporcionalidade entre a dureza e a densidade básica de diferentes espécies amazônicas.

Kollmann e Coté Jr (1968) discorrem sobre as relações diretas entre estas propriedades e estabeleceram classificações para os níveis de dureza das espécies de acordo com intervalos de densidade. Desse modo, a dureza, assim como as outras propriedades mecânicas da madeira, é influenciada pela densidade da madeira e, no caso da madeira de galho e fuste das espécies testadas, estas são classificadas 
como de alta densidade, configurando elevada resistência à penetração tanto na direção normal ou paralela às fibras, com aptidão para o seu emprego em pisos em geral, desde que verificadas as demais propriedades físicas como sua estabilidade dimensional.

A diferença entre os valores de resistência mecânica obtidos para galho e fuste das espécies ou a distanciação em relação ao encontrados em literatura pode estar atrelada às diferentes posições de obtenção dos corpos de prova no cerne, à idade das árvores amostradas, à espécie avaliada dentro do grupo de espécies agrupadas no mesmo gênero e, principalmente, a densidade da madeira comparada.

Os resultados obtidos para os coeficientes de variação estão em conformidade com a NBR 7190 (1997) a qual admite o limite de $18 \%$ para a resistência à esforços normais e $28 \%$ para a resistência a esforços tangenciais. As madeiras provenientes de galho das espécies estudadas se enquadram na classe de resistência C60 de acordo com a NBR 7190 (1997), a qual estabelece valores mínimos de resistência à compressão paralela às fibras de 60 $\mathrm{MPa}$, para a resistência ao cisalhamento de 8 MPa e densidade básica de 0,80 g $\mathrm{cm}^{-3}$ a $12 \%$ de umidade.

O uso de classes de resistência para a madeira viabiliza o emprego da espécie madeireira que se encontra disponível, neste caso a madeira de resíduos florestais apta ao desdobro. Se mostra necessário estudos que avaliem as particularidades da madeira de galho e analisem aspectos estruturais, como o valor característico, para viabilizar e certificar o emprego desse material em projetos estruturais de maiores dimensões.

\section{CONCLUSÃO}

A densidade básica da madeira de resíduos florestais das espécies estudadas de 0,81 $\mathrm{g} \mathrm{cm}^{-3}$ para Manilkara sp. e 0,91 $\mathrm{g} \mathrm{cm}^{-3}$ para a madeira de galho de Hymenaea sp. sendo são classificadas como de densidade alta.

O comportamento mecânico da madeira de galhos foi semelhantes à da 
base do fuste em termos gerais e, se tratando de espécies de estimado valor comercial, a utilização desse material caracteriza uma atividade com potencial para geração de produtos com valor agregado, desde que consideradas suas particularidades como seu diâmetro ou posição de obtenção da peça no cerne do galho e, principalmente, sua estabilidade dimensional.

\section{AGRADECIMENTOS}

Ao Conselho Nacional de Desenvolvimento Científico e Tecnológico - CNPq pela concessão da bolsa de fomento, à Rondobel Florestal por viabilizar a obtenção do material e ao Laboratório de Tecnologia da Madeira - LTM pelo apoio para a realização da pesquisa.

\section{REFERÊNCIAS}

ABNT - Associação Brasileira de Normas Técnicas. NBR 7190: Projeto de estruturas de madeira. Rio de Janeiro, 1997. 107p.

ALMEIDA, D. H.; SCALIANTE, R. M.; MACEDO, L. B.; MACEDO, A. N.; DIAS,
A. A.; CHRISTOFORO, A. L.; JUNIOR, C. C. Caracterização completa da madeira da espécie amazônica Paricá (Schizolobium amazonicum Herb) em peças de dimensões estruturais. Revista Árvore, Viçosa-MG, v. 37, n. 6, p. 11751181, 2013.

ARAÚJO, H. J. B. Relações funcionais entre propriedades físicas e mecânicas de madeiras tropicais brasileiras. Floresta, Curitiba, v. 37, n. 3, p. 7, set/dez. 2007.

AMERICAN SOCIETY FOR TESTING AND MATERIALS. ASTM. ASTM D143 14 - Standard Test Methods for Small Clear Specimens of Timber. In: Annual book of ASTM Standards, 2009.

BAGGIO, A. J.; CARPANEZZI, A. A. Quantificação dos resíduos florestais em bracatingais na região metropolitana de Curitiba, PR. Boletim Pesquisa Florestal, v. 30/31, p. 51-66, 1995.

BARBOSA, A. P.; VIANEZ, B. F.; VAREJÃO, M. J.; ABREU, R. L. S. Considerações sobre o perfil tecnológico do setor madeireiro na Amazônia Central. Parcerias estratégicas, Brasília, n. 12, p. 42-61, set. 2001.

BATISTA, D. C.; KLITZKE, R. J.; SANTOS, C. V. T. Densidade básica e retratibilidade da madeira de clones de três espécies de Eucalyptus. Ciência Florestal, Santa Maria, v. 20, n. 4, p. 665-674, out-dez., 2010. 
BELTRAME, R.; SOUZA, J. T.; MACHADO, W. G.; VIVIAN, M. A.; BULIGON, E. A.; PAULESKI, D. T.; GATTO, D. A.; HASELEIN, C. R. Propriedades físico-mecânicas da madeira de Araucaria angustifolia (Bertol.) em três estratos fitossociológicos. Ciência da Madeira, Pelotas, v. 01, n. 02, p. 54-69, 2010.

BERGMAN, R.; CAI, Z.; CARLL, C. G.; CLAUSEN, C. A.; DIETENBERGER, M. A.; FALK, R. H. FRIHART, C. R.; GLASS, S. V.; HUNT, C. G.; IBACH, R. E.; KRETSCHMANN, D. E.; RAMMER, D. R.; ROSS, R. J. Forest Products Laboratory. Wood handbook - Wood as an engineering material. General Technical Report FPL-GTR-190. Madison, WI: U.S.: Department of Agriculture, Forest Service, Forest Products Laboratory, 2010. 508 p.

BRAZ, R. L.; NUTTO, L.; BRUNSMEIER, M.; BECKER, G.; SILVA, D. A. Resíduos da colheita florestal e do processamento da madeira na Amazônia - uma análise da cadeia produtiva. Journal of Biotechnology and Biodiversity, v. 5, n. 2, p. 168-181, 2014.

CARDOSO, C.C. Caracterização tecnológica das espécies de maior frequência no segundo ciclo de corte na Floresta Nacional do Tapajós, Piracicaba, 2015. 92f. Dissertação (Mestrado em Recursos Florestais) Escola Superior de Agricultura "Luiz de Queiroz", Piracicaba, 2015.

DIAS, F. M.; LAHR, F. A. R. Estimativa de propriedades de resistência e rigidez da madeira através da densidade aparente. Revista Scientia Forestalis, n. 65, p. 102-113, Piracicaba, 2004.

DUTRA, R. I. J. P.; NASCIMENTO, S. M.; NUMAZAWA, S. Resíduos de Indústria madeireira:

Caracterização, consequências sobre o meio Ambiente e opções de uso. Revista Científica Eletrônica de Engenharia Florestal, n. 5, p. 19, 2005.

EVANS, J. L. W.; SENFT, J. F.; GREEN, D. $W$. Juvenile wood effect in red alder: analysis of physical and mechanical data to delineate juvenile and mature wood zones. Forest Products Journal, v. 50, n. 7/8, p. 75-87, 2000.

FEITOSA, B. C. Aproveitamento dos resíduos de madeira no Pará. Revista da Madeira, v. 13, n. 114, p. 1, 2008.

GOMES, J. I.; SILVA, E. M. A.; MELO, A. T. S. Durabilidade de 15 espécies de madeiras amazônicas em contato com o solo em ambiente sombreado. Belém: EMBRAPA, 2005. (Comunicado Técnico, n. 148).

GONÇALEZ, J. C.; ZERBINI, N. J. ; JANIN, G. Caractérisation technologique des bois amazoniens immergés dans le barrage de Tucuruí au Brésil. Bois et Forêts des Tropiques, Paris, França, v. 274, n. 274, p. 45-56, 2002.

GONÇALVES, F. G.; OLIVEIRA, J. T. S.; LUCIA, R. M. D.; NAPPO, M. E.; SARTÓRIO, R. C. Densidade básica e variação dimensional de um híbrido clonal de Eucalyptus urophylla $\mathrm{x}$ 
Eucalyptus grandis. Revista Árvore, Viçosa - MG, v. 33, n. 2, p. 277-288, 2009a.

GONÇALVES, J. E.; SARTORI, M. M. P.; LEÃO, A. L. Energia de briquetes produzidos com rejeitos de resíduos sólidos urbanos e madeira de Eucalyptus grandis. Revista Brasileira de Engenharia Agrícola e Ambiental, v. 13, n. 5, p. 657-661, 2009b.

GONÇALVEZ, F. G.; LELIS, R. C. C. Caracterização tecnológica da madeira de Acacia mangium Willd em plantio consorciado com Eucalipto. Floresta e Ambiente, v. 19, n. 3, p. 286-295, jul./set. 2012.

IDEFLOR - Instituto de Desenvolvimento Florestal do Estado do Pará. Relatório ambiental preliminar das áreas de concessão florestal do conjunto de glebas Mamurú-Arapiuns. Relatório Final. p. 16. Belém, 2010.

IPT - Instituto de Pesquisas Tecnológicas do Estado de São Paulo. Catálogos de madeiras para construção civil. São Paulo: IPT: Instituto de Pesquisas Tecnológicas do Estado de São Paulo. 106p, 2013.

KANASHIRO, M. O manejo florestal o manejo florestal e a promoção da gestão dos recursos florestais em áreas de uso comunitário e familiar na Amazônia. Cadernos de Ciência \& Tecnologia, Brasília, v. 31, n. 2, p. 421427, maio/ago. 2014.

KOLLMANN, F. F. P.; CÔTÉ JR, W. A. Principles of wood science and technology: solid wood. Berlin: Springer-Verlag, 1968. v. 1, 592 p.

LAHR, F. A. R.; CHAHUD, E.; FERNANDES, R. A; TEIXEIRA, R. S. Influência da densidade na dureza paralela e na dureza normal às fibras para algumas espécies tropicais brasileiras. Scientia Forestalis, Piracicaba, v. 38, n. 86, p. 153-158, jun. 2010.

LAHR, F. A. R.; CHRISTOFORO, A. L.; Silva, C. E. G.; JUNIOR, J. R. A.; PINHEIRO, R. V. Avaliação de propriedades físicas e mecânicas de madeiras de jatobá (Hymenaea stilbocarpa Hayne) com diferentes teores de umidade e extraídas de regiões distintas. Revista Árvore, v. 40, n. 1, p. 147-154, Viçosa - MG, 2016.

LATORRACA, J. V. F.; ALBUQUERQUE, C. E. C. Efeito do rápido crescimento sobre as propriedades da madeira. Floresta e Ambiente, v. 7, n. 1, p. 279291, 2000.

LEONELLO, E. C.; BALLARIN, A. W.; OHTO, J. M.; PALMA, H. A. L.; ESCOBAR, J.F. Classificação Estrutural e Qualidade da Madeira do Clone GT 1 de Hevea brasiliensis Muell. Arg. Floresta e Ambiente, v. 19, n. 2, p. 229235, 2012.

LOBÃO, M. S.; LÚCIA, R. M. D.; MOREIRA, S. S. S.; GOMES, A. Caracterização das propriedades físico-mecânicas da madeira de eucalipto com diferentes densidades. Revista Árvore, v. 28, n. 6, p. 889-894, 2004. 
LOGSDON, N. B.; FINGER, Z.; ROSA, L. M. Caracterização da madeira de Vochysia guianensis Aubl. Engenharia Civil, n. 29, p. 57-69, 2007.

MIRANDA, M. C.; CASTELO, P. A. R.; MIRANDA, D. L. C.; Rondon, E.V. Propriedades físicas e mecânicas da madeira de Parkia gigantocarpa Ducke. Ciência da Madeira, Pelotas, v. 3, n. 2, p. 55-65, 2012.

OLIVEIRA, J. T. S; SILVA, J. C. Variação radial da retratibilidade e densidade básica da madeira de Eucalyptus saligna Sm. Revista Árvore, Viçosa-MG, v. 27, n. 3, p. 381-385, 2003.

PEREIRA, F. A.; SANTOS, R.; CAMPOS, C. I.; MOLINA, J. C. Utilização de resíduos e derivados de madeira para confecção de uma guitarra elétrica. Scientia Forestalis, Piracicaba, v. 39, n. 90, p. 183-190, jun. 2011.

PIGOZZO, R. J. B. Aplicabilidade do infravermelho-próximo (NIR) na identificação e estimativa de propriedades da madeira. Madeira, arquitetura e engenharia. v. 10, n. 25, p. 59-61, 2009.

PONTES, S. M. A.; AFONSO, D. G.; NASCIMENTO, D. L. Análise comparativa das Propriedades Físicomecânicas da madeira de galho e fuste de 2 espécies florestais com potencial madeireiro. Anais... $4^{\circ}$ Congresso Florestal Paranaense. p. 5, 2012.

PULITO, A. P.; JÚNIOR, J. C. A. Manejo de Resíduos Florestais. Revista
Opiniões. v. 4, p. 5. Dez. 2008 - Fev. 2009.

ROSA, R. A.; FRANÇA, L. C. A; SEGUNDINHO, P. G. A.; LUBE, V. M.; PAES, J. B. Caracterização da madeira de maçaranduba (Manilkara sp.) por métodos destrutivos e não destrutivos. Ciência da Madeira, Pelotas, v. 05, n. 01, p. 68-78, 2014.

SANTOS, S. R.; SANSígOlO, C. A. Influência da densidade básica da madeira de clones de Eucalyptus grandis $x$ Eucalyptus urophylla na qualidade da polpa branqueada. Ciência Florestal, Santa Maria, v. 17, n. 1, p. 53-63, jan-mar, 2007.

SECRETARIA DE ESTADO DE MEIO AMBIENTE. SEMAS. Extração e Movimentação de Tora de Madeiras Nativas por município. Relatórios anuais, 2006-2015. 2016.

SETTE, J. R.; OLIVEIRA, C. R.; TOMAZELLO FILHO, I. R.; M.; YAMAJI, F. M.; LACLAU, J. P. Efeito da idade e posição de amostragem na densidade e características anatômicas da madeira de Eucalyptus grandis. Revista Árvore, Viçosa-MG, v. 36, n. 6, p. 11831190, 2012.

SERVIÇO FLORESTAL BRASILEIRO. SFB. Espécies madeireiras de interesse comercial. Laboratório de Produtos Florestais (adaptado). 2016. Disponível em:

http://www.florestal.gov.br/snif/recurs os-florestais/especies-florestais.

Acesso em: 20 de setembro de 2017. 
SILVA FILHO, D. F.; ROCHA, J. S.; MOURA, J. B. Influência da densidade na dureza Janka em oito espécies madeireiras da Amazônia Central. Acta Amazonica, vol. 22. n. 2. p. 275 - 283, Manaus, 1992.

SILVA-RIBEIRO, R. B. Quantificação e valoração de resíduos da colheita florestal na Floresta Nacional do Tapajós, Pará. 90f. Viçosa, 2013. Dissertação (Mestrado em Ciências Florestais) - Universidade Federal de Viçosa, Viçosa, 2013.

SILVEIRA, L. H. C.; REZENDE, A. B.; VALE, A. T. Teor de umidade e densidade básica da madeira de nove espécies comerciais amazônicas. Acta Amazonica, v. 43, n. 2, p. 179 - 184, 2013.

SISFLORA. Sistema de Comercialização e Transporte de Produtos Florestais. Disponível em: http://monitoramento.semas.pa.gov.br /sisflora/. 2013. Portal SISFLORA - PA.

SOUZA, M. H.; MAGLIANO, M. M.; CAMARGOS, J. A. A.; SOUZA, M. R. Madeiras topicais brasileiras. 2a ed. Brasília: LPF/ IBAMA, 2002, 152p.

ZAU, M. D. L.; PEREIRA, R. V.; GIACON, V. M.; LAHR, F. A. R. Avaliação das propriedades química, física e mecânica de painéis aglomerados produzidos com resíduo de madeira da Amazônia - Cumaru (Dipteryx odorata) e resina poliuretana à base de óleo de mamona. Polímeros: Ciência e Tecnologia, v. 24, n. 6, p. 726-732, 2014. 